\section{Exposição ocupacional à sílica no Brasil no ano de 2001}

\section{Occupational exposure to silica in Brazil in 2001}

Fátima Sueli Neto Ribeiro'

Esther Archer de Camargo ${ }^{2}$

Eduardo Algranti ${ }^{3}$

Victor Wünsch Filho ${ }^{4}$

${ }^{1}$ Universidade do Estado do Rio de Janeiro \& Instituto Nacional do Câncer

2 Departamento de Enfermagem da Universidade de Federal de São Paulo

${ }^{3}$ Divisão Medicina do Centro Técnico Nacional do Fundacentro

${ }^{4}$ Departamento de Epidemiologia da Faculdade Saúde Pública da Universidade de São Paulo

Agradecimentos: Os autores agradecem pelo apoio aos pesquisadores, técnicos e sindicalistas que generosamente contribuíram com informações, realizaram as classificações e auxiliaram na análise deste trabalho, bem como à equipe de suporte da DATAMEC, ao Laboratório de Epidemiologia e Estatística do Instituto Dante Pazzanese de Cardiologia e à Fundacentro. Correspondência: Fátima Sueli Neto Ribeiro. UERJ/INU - Rua São Francisco Xavier 524, Bloco D, sala 12002 , Rio de Janeiro,RJ, CEP 20.550-900.E-mail: fatsue@uerj.br; fatsuerj@gmail.com

\section{Resumo}

Objetivo: Estimar o número de trabalhadores brasileiros expostos à sílica no ano de 2001. Método: Informações sobre ocupações e setores econômicos foram reunidas em uma matriz de exposição ocupacional (MEO) com 347 categorias ocupacionais por 25 subsetores econômicos. Informações sobre o número de trabalhadores por ocupação foram extraídas da base de dados Relatório Anual de Informações Sociais (RAIS) do Ministério do Trabalho e Emprego. A exposição à sílica foi avaliada e classificada por dois peritos em quatro categorias, de acordo com a freqüência semanal de exposição no ambiente de trabalho. Resultados: Foram considerados não expostos 31.451 .594 trabalhadores $(85,7 \%)$, possivelmente expostos 976.939 (2,65\%), provavelmente expostos 2.404 .955 (6,52\%) e definitivamente expostos à sílica 2.065 .929 (5,6\%). Os setores com a maior prevalência de exposição foram: construção civil $65 \%$, extração de pedras 59\%, indústria de mineral não metálico $55 \%$ e indústria metalúrgica $24 \%$. No setor de serviços de terceiros, a prevalência foi de $2 \%$. Conclusão: A prevalência de trabalhadores brasileiros definitivamente expostos à sílica é mais alta do que aquela observada em países europeus, onde estudos semelhantes foram conduzidos.

Palavras-chaves: Sílica. Matriz de exposição ocupacional. Epidemiologia. Exposição ocupacional.

O estudo foi dispensado de apreciação no Comitê da Faculdade de Saúde Pública/USP em 2002. 
Abstract

Objective: To estimate the number of Brazilian workers exposed to silica in 2001. Methods: Information on occupation and industry for the year 2001 was linked through a job exposure matrix (JEM) of 347 job categories in 25 industries. Data on the number of workers were extracted from the National Workforce Database of the Ministry of Labor and Employment. Two experts classified silica exposure according to frequency in four categories, according to weekly proportion of exposure to silica in the work environment. Results: 31,451,594 (85.7\%) workers were classified as not exposed; 976,939 (2.65\%) as possibly exposed; $2,404,955$ (6.52\%) as probably exposed; and $2,065,929(5.6 \%)$ as definitely exposed to silica. Industries with a higher prevalence were: construction $65 \%$, quarrying $59 \%$, manufacturing of non metallic mineral products $55 \%$, manufacturing of basic metals $24 \%$, and rent of machinery and other business activities $2 \%$. Conclusions: The prevalence of Brazilian workers definitely exposed to silica is higher than the one observed in European countries, where similar studies were conducted.

Keywords: Silica. Job exposure matrix. Epidemiology. Occupational exposure.

\section{Introdução}

A exposição ocupacional à sílica está relacionada com a silicose e a bronquite crônica, e também, com a maior incidência de tuberculose e de algumas doenças auto-imunes. A associação da sílica com câncer foi objeto de intenso debate nos anos oitenta ${ }^{1}$. Desde 1997, a Agência Internacional para Pesquisa em Câncer (IARC) classifica a sílica como substância definitivamente cancerígena para humanos ${ }^{2}$. Portanto, a exposição ocupacional à sílica caracteriza um problema de saúde pública que exige melhor conhecimento para o planejamento de adequadas intervenções.

A sílica cristalina constitui mais de $80 \%$ da crosta da terra e é usada de forma intensiva em vários processos de trabalho. A exposição à sílica tem sido descrita na mineração, extração e beneficiamento de pedras, construção civil, fundição e fabricação de vidros e cerâmica ${ }^{2}$, além de estar presente em diversas fases da produção de setores econômicos variados, como na indústria de alimentos e bebida, indústria de madeira, produtos de borracha, agricultura, produção de petróleo cru, realização de serviços dentários e outros ${ }^{3}$. No Brasil, a dimensão da exposição ocupacional à sílica é virtualmente desconhecida, pois não existe nenhum inquérito de avaliação ambiental que permita a configuração de um cenário da situação nacional. As estimativas com base em projeções de população ocupada dimensionam em aproximadamente seis milhões o número de trabalhadores expostos ${ }^{4}$.

A importância da silicose no Brasil tem sido descrita desde 1939 e, atualmente, é a pneumoconiose com maior prevalência no país ${ }^{5}$. Entretanto, sua dimensão não é completamente conhecida. Estudos recentes permitem aproximações pontuais sobre a ocorrência da doença em algumas atividades industriais. Altas prevalências de silicose (categoria radiológica $1 / 1$ ou maior, de acordo com a classificação da Organização Internacional do Trabalho - OIT) foram encontradas em trabalhadores da 
indústria de construção naval $(23,6 \%)^{6}$ e em cavadores de poços artesianos $(17,4 \%)^{7}$. Prevalências entre entre 3 e 5\% foram encontradas nos escultores de pedra ${ }^{8}$, na indústria de cerâmica ${ }^{9}$ e nas fundições ${ }^{10}$. Os registros oficiais da Previdência Social, mesmo considerando-se a alta subnotificação, revelam a ocorrência de dois casos de silicose para 10.000 contribuintes no ano de $2003^{11}$.

O objetivo deste trabalho foi estimar o número de trabalhadores expostos à sílica no Brasil no ano de 2001, e identificar as atividades econômicas onde essa exposição é mais importante, propiciando assim informações para a implantação de medidas adequadas de prevenção.

\section{Material e Método}

A população brasileira economicamente ativa (PEA) foi dimensionada em 76.158.531 trabalhadores pelo censo demográfico de $2000^{12}$. A base de dados RAIS (Relatório Anual de Informações Sociais), do Ministério do Trabalho e Emprego, registra aqueles vinculados ao sistema de CLT (Consolidação de Leis Trabalhistas) e no ano de 2001 assinalava aproximadamente $47 \%$ destes trabalhadores. Foram considerados 36.899.417 trabalhadores de ambos os sexos na base RAIS como tendo informação suficiente para fins deste estudo. A pertinência do uso desta base para fins de estudo em saúde do trabalhador foi discutida em publicação anterior ${ }^{13}$. Não foram discriminadas diferenças entre sexos, embora haja consistentes indicações de que a maioria dos expostos é constituída de homens.

A variável ocupação foi agrupada de acordo com a Classificação Brasileira de Ocupações (CBO), de 1995, com discriminação de três dígitos, resultando em 347 categorias. Esta classificação corresponde à Classificação Padrão Internacional de Ocupações (International Standard Classification of Occupations - ISCO). O setor econômico foi agrupado de acordo com a Classificação Nacional de Atividades Econômicas (CNAE),
1994, que é compatível com a Classificação Padrão Internacional de Atividades Econômicas (Industrial Standard Internacional Classification - ISIC). O detalhamento em dois dígitos resultou em 25 setores econômicos que incluem indústrias, serviços, agricultura, extração e comércio.

\section{Avaliação de Exposição}

A exposição ocupacional à sílica foi definida como a situação em que o trabalhador está submetido à exposição à sílica cristalina livre no seu processo de trabalho. Para a avaliação da exposição foi desenvolvida uma Matriz de Exposição Ocupacional (MEO) de dupla entrada construída a partir do cruzamento da informação de ocupação para cada setor econômico. A exposição ocupacional foi estimada por um epidemiologista e um higienista ocupacional pela análise das 8.675 células resultantes da intersecção das duas variáveis. A exposição foi codificada segundo a freqüência semanal potencial de exposição em quatro grupos, utilizando-se critério semelhante ao proposto por Siemiatycki $(1991)^{14}$ : não exposto (NE), exposição estimada igual ou menor a observada na população geral, ou exposições maiores, mas até $1 \%$ da jornada semanal de trabalho; possivelmente exposto (PsE), atividades profissionais descritas muito genericamente, mas podem resultar em exposições de 1 a $5 \%$ da jornada de trabalho semanal; provavelmente exposto (PrE), exposição estimada como mais alta do que na população geral, mas com grande variação entre empresas. A descrição da ocupação pela CBO permite predizer exposições esporádicas entre 5 e $30 \%$ da jornada semanal de trabalho; definitivamente exposto (DE), exposição estimada considerada como freqüente, maior que $30 \%$ da jornada semanal de trabalho, e a informação disponível foi considerada confiável. Detalhes da construção e estudo de validação desta matriz encontram-se em publicação prévia ${ }^{15}$.

A codificação levou em consideração atividades consideradas tipicamente com exposição à sílica; informações de indústrias, 
da literatura internacional e nacional; entrevistas com profissionais de empresas de setores industriais específicos e com sindicalistas, além da experiência pessoal dos codificadores. A estrutura da MEO seguiu o mesmo formato tabular da RAIS. Assim, foi possível sua justaposição à tabulação da freqüência absoluta de trabalhadores no ano 2001, calculada a partir da RAIS em planilhas gerada para o software Excell ${ }^{\circledR}$. Finalmente, foi realizada uma análise de consistência da classificação por especialistas externos cegos para a classificação anterior.

\section{Resultados}

Foram identificados $14,8 \%$ trabalhadores com algum nível de exposição para sílica: destes foram identificados $2,7 \%$ possivelmente expostos, $6,5 \%$ provavelmente expostos e 5,6\% definitivamente expostos (Tabela 1).

Tabela 1 - Número e porcentagem de trabalhadores expostos e não-expostos à sílica em todos os setores da economia por categorias de exposição. Brasil, 2001.

Table 1 - Number and percentage of workers exposed and not exposed to silica in all economic segments by exposure category. Brazil, 2001

\begin{tabular}{lcc}
\hline $\begin{array}{l}\text { Classificação da } \\
\text { exposição }\end{array}$ & $\mathrm{N}$ & $\%$ \\
\hline $\begin{array}{l}\text { Não Exposto } \\
\text { Possivelmente }\end{array}$ & $\begin{array}{c}31.451 .594 \\
976.939\end{array}$ & $\begin{array}{c}85,2 \\
2,7\end{array}$ \\
$\begin{array}{l}\text { Exposto } \\
\text { Provavelmente }\end{array}$ & 2.404 .955 & 6,5 \\
$\begin{array}{l}\text { Exposto } \\
\text { Definitivamente }\end{array}$ & 2.065 .929 & 5,6 \\
$\begin{array}{l}\text { Exposto } \\
\text { Total }\end{array}$ & 36.899 .417 & 100 \\
\hline
\end{tabular}

'De acordo com a matriz de exposição ocupacional (MEO) utilizada.

${ }^{1}$ According to the job exposure matrix (JEM) used

Considerando-se o número de trabalhadores ocupados em cada setor econômico, os setores econômicos que apresentaram as proporções mais altas de expos- tos foram: construção civil, extração mineral e pedreiras, indústria de mineral nãometálico e metalurgia. No setor de construção civil a proporção de trabalhadores definitivamente expostos para sílica alcançou 65\% (Tabela 2).

A prevalência de trabalhadores definitivamente expostos à sílica no Brasil é comparativamente maior do que em países europeus que utilizaram metodologia de estimação semelhante no contexto do projeto CAREX ${ }^{3}$ (Tabela 3).

\section{Discussão}

O uso de matrizes de exposição ocupacional (MEO) tem sido implementado em vários países ${ }^{16-18}$. $\mathrm{O}$ alto nível de concordância entre o julgamento de especialistas, discutido por Rybicki et al. (1997) ${ }^{19}$, justifica a utilização deste método para estimar exposições ocupacionais.

A aplicação da MEO neste estudo identificou uma prevalência de 5,6\% trabalhadores brasileiros definitivamente expostos à sílica, o que excede ao observado em países da Comunidade Européia, onde foi utilizado um método de estimação da exposição semelhante a este - o Sistema de Avaliação para Exposição Ocupacional a Cancerígenos (Evaluation System to Carcinogen of Occupational Exposure CAREX), que identificou em diferentes países daquela região exposições entre trabalhadores no mercado formal da ordem de $0,5 \%$ a $3,9 \%^{17}$. A maior prevalência observada no Brasil pode estar associada às precárias condições de segurança e higiene no Brasil, que agravaria os riscos de exposição, mas, também, pode ser uma decorrência das peculiaridades dos métodos de avaliação da exposição entre os estudos.

$\mathrm{Na}$ construção da presente MEO, a prevalência da exposição foi estimada a partir da análise de distintas ocupações por diferentes setores econômicos. Para a classificação da exposição, os especialistas consideraram as reais condições brasileiras, tanto do ponto de vista dos processos produtivos como de saúde e segurança nas 
Tabela 2 - Número e porcentagem de trabalhadores não-expostos e expostos à sílica em vários níveis, de acordo com setor econômico. Brasil, 2001

Table 2 - Number and percentage of workers exposed and not exposed to silica at several levels, according to economic segment. Brazil, 2001

\begin{tabular}{|c|c|c|c|c|c|c|c|c|}
\hline \multirow[t]{2}{*}{ Setor Econômico } & \multicolumn{2}{|c|}{ Não Expostos } & \multicolumn{2}{|c|}{$\begin{array}{l}\text { Possivelmente } \\
\text { Expostos }\end{array}$} & \multicolumn{2}{|c|}{$\begin{array}{c}\text { Provavelmente } \\
\text { Expostos }\end{array}$} & \multicolumn{2}{|c|}{$\begin{array}{c}\text { Definitivamente } \\
\text { Expostos }\end{array}$} \\
\hline & $\mathrm{n}$ & $\%$ & $\mathrm{n}$ & $\%$ & $\mathrm{n}$ & $\%$ & $\mathrm{n}$ & $\%$ \\
\hline Construção & 533208 & 23,93 & 145319 & 6,52 & 101434 & 4,55 & 1447898 & 64,99 \\
\hline Extração Mineral & 25396 & 17,23 & 26564 & 18,03 & 8398 & 5,70 & 86995 & 59,04 \\
\hline Indústria de Mineral não Metálico & 54872 & 14,79 & 81006 & 21,84 & 30699 & 8,28 & 204327 & 55,09 \\
\hline Metalurgia & 382338 & 58,46 & 96521 & 14,76 & 18262 & 2,79 & 156877 & 23,99 \\
\hline Agricultura & 554556 & 26,99 & 77422 & 3,77 & 1347313 & 65,57 & 75566 & 3,67 \\
\hline Indústria Borracha & 270462 & 85,09 & 5550 & 1,74 & 33490 & 10,53 & 8387 & 2,64 \\
\hline Prestação de Serviço & 3491059 & 81,25 & 243712 & 5,67 & 489921 & 11,40 & 72027 & 1,68 \\
\hline Indústria Eletrônica & 228040 & 87,74 & 28700 & 11,04 & 1707 & 0,66 & 1465 & 0,56 \\
\hline Indústria Mecânica & 327673 & 85,13 & 11189 & 2,91 & 44258 & 1,15 & 1781 & 0,46 \\
\hline Indústria Química & 655443 & 96,45 & 8062 & 1,19 & 13754 & 2,02 & 2304 & 0,34 \\
\hline Indústria Material Transporte & 230206 & 62,73 & 60622 & 16,52 & 75366 & 20,54 & 780 & 0,21 \\
\hline Ind Alimento e Bebidas & 1481222 & 98,52 & 917 & 0,06 & 19153 & 1,27 & 2184 & 0,15 \\
\hline Comércio Atacadista & 1014563 & 98,47 & 5297 & 0,51 & 9656 & 0,94 & 865 & 0,08 \\
\hline Comércio Varejista & 5394842 & 97,93 & 62465 & 1,13 & 47568 & 0,86 & 3752 & 0,07 \\
\hline Serviço Público & 304957 & 86,98 & 33732 & 9,62 & 11738 & 3,35 & 171 & 0,05 \\
\hline Indústria Madeira & 542146 & 97,21 & 11702 & 2,10 & 3680 & 0,66 & 156 & 0,03 \\
\hline Ind Têxtil & 951747 & 95,70 & 39944 & 4,02 & 2685 & 0,27 & 110 & 0,01 \\
\hline Transporte e Comunicação & 1875124 & 98,27 & 25012 & 1,31 & 8063 & 0,42 & 55 & 0,00 \\
\hline Hotelaria & 3328175 & 98,96 & 2441 & 0,07 & 32408 & 0,97 & 114 & 0,00 \\
\hline Ensino & 1109805 & 98,97 & 735 & 0,07 & 10769 & 0,96 & 39 & 0,00 \\
\hline Administração Pública & 6053410 & 98,56 & 4812 & 0,08 & 83676 & 1,36 & 76 & 0,00 \\
\hline Indústria de Papel e Gráfica & 396391 & 98,59 & 4047 & 1,01 & 1596 & 0,40 & - & - \\
\hline Indústria de Calçado & 391685 & 99,88 & 251 & 0,06 & 247 & 0,06 & - & - \\
\hline Setor Financeiro & 701440 & 99,89 & 32 & 0,00 & 740 & 0,11 & - & - \\
\hline Serviço Médico & 1152834 & 99,20 & 885 & 0,08 & 8374 & 0,72 & - & - \\
\hline Total & 31451594 & 85,24 & 976939 & 2,65 & 2404955 & 6,52 & 2065929 & 5,60 \\
\hline
\end{tabular}

empresas. Portanto, com resultados muito próximos à realidade brasileira. Diferentemente, o CAREX utilizou como referência prevalências de exposição estimadas em dois países: Finlândia e Estados Unidos. Estas estimativas foram ajustadas à estrutura econômica de cada país, sem que fosse levada em consideração, inicialmente, a especificidade do padrão de exposição de cada país. Em alguns países especialistas locais procederam as correções das estimativas ${ }^{20}$.

As limitações deste estudo incluem um possível viés de informação, que pode ter ocorrido a partir da análise das informações, apenas levando em conta o título da ocupação. Reconhece-se que ocorre alta variabilidade de exposição nos mesmos grupos ocupacionais ${ }^{21}$. Por outro lado, o banco de dados RAIS possui característica administrativa e espontânea ${ }^{22}$ e não conta com supervisão e controle de qualidade da informação prestada. Outra possível limitação está relacionada à descrição da ocupação, que pode não ter expressado todo o conjunto de atividades que são executadas pelo trabalhador. Nestas situações, a experiência dos peritos foi fundamental para as estimativas de exposição. Finalmente, este estudo é restrito a trabalhadores formais e empregados, ou seja, cerca de $40 \%$ da população economicamente 
Tabela 3 - Comparação entre trabalhadores expostos à sílica no Brasil [2001] e em outros países selecionados da base de dados CAREX'.

Table 3 - Comparison between workers exposed to silica in Brazil [2001] and in other countries selected from the CAREX database

\begin{tabular}{lccc}
\hline País & $\begin{array}{c}\text { Trabalhadores } \\
\text { Ocupados } \\
\text { Milhares }\end{array}$ & \multicolumn{2}{c}{ Trabalhadores } \\
Expostos \\
Brasil & 35.070 & 1.841 & 5,6 \\
Finlândia & 2.125 & 82 & 3,9 \\
República Tcheca & 5.000 & 170 & 3,4 \\
Espanha & 12.400 & 400 & 3,2 \\
Alemanha & 34.583 & 1.000 & 2,9 \\
Grã Bretanha & 22.727 & 590 & 2,6 \\
Portugal & 4.041 & 83 & 2,1 \\
Itália & 17.500 & 280 & 1,6 \\
França & 21.304 & 110 & 0,5 \\
\hline
\end{tabular}

${ }^{1}$ Referência 17.

${ }^{2}$ Classificados como definitivamente expostos à sílica.

${ }^{1}$ Reference 17.

${ }^{2}$ Classified as definitely exposed to silica

ativa. Os trabalhadores não-formais, artesãos, autônomos, trabalhadores em indústria em pequena escala e inativos estão excluídos dos cálculos. Muitos destes trabalhadores podem estar executando atividades com exposições importantes, como os cavadores de poços, extração e beneficiamento de pedras decorativas, pequenos agricultores, entre tantos outros.

Considerando-se todos os níveis de exposição, os setores que apresentaram freqüência mais alta de trabalhadores expostos à sílica são semelhantes aos descritos em estudos e compilações de referência $^{2}$, com destaque para a construção civil; agricultura; indústria de material não metálico, particularmente cerâmica e vidro; construção e reparo naval; e fundição. $\mathrm{O}$ importante papel do setor de serviços (aluguel de maquinaria e mão de obra) no Brasil resultou em aproximadamente $2 \%$ de trabalhadores definitivamente expostos, $11 \%$ provavelmente expostos e $6 \%$ possivelmente expostos. Esta situação pode ser compreendida pela dinâmica da força de trabalho brasileira que têm aumentado no setor de serviço, quando comparadas ao setor industrial. Assim, uma proporção ainda maior de trabalhadores do setor de serviço pode estar exposta por mais que $30 \%$ da jornada semanal de trabalho, dependendo do setor econômico examinado. A mobilidade e as condições desfavoráveis de proteção à saúde desses trabalhadores terceirizados, reiteradamente descritos, pode estar gerando um perfil complexo de exposição a um crescente número de agentes, alguns potencialmente carcinogênicos.

Informações sobre a dimensão da exposição de trabalhadores brasileiros expostos à sílica são escassas. Estudo em pedreiras demonstrou a ocorrência de alto nível de exposição em todas as tarefas relacionadas à atividade de extração e trituração da rocha ${ }^{23}$. O mesmo foi observado na indústria da cerâmica, especialmente no acabamento, vitrificação, limpeza com ar comprimido, estamparia, refratários e forno, onde altos níveis de exposição foram identificados em todas as ocupações ${ }^{23}$ e na construção civil ${ }^{24}$.

Nos Estados Unidos, relatos da agência responsável pela fiscalização das operações de mineração, MSHA (Mine Safety and Health Administration) e do NIOSH 
(National Institute for Occupational Safety and Health), revelaram que, em mais de $80 \%$ das empresas fiscalizadas, o nível de exposição à sílica excedia o limite de tolerância estabelecida pela legislação vigen$\mathrm{te}^{25}$. Embora não existam informações sistematizadas no Brasil, relatos de técnicos dos órgãos públicos brasileiros indicam que a proporção pode ser ainda maior.

A redução progressiva dos níveis de exposição é uma das estratégias a ser adotada num processo contínuo de vigilância da saúde, em contraposição à utilização de parâmetros de limites de exposição que, além de não serem respeitados, induzem à aceitação de riscos potenciais consideráveis de câncer e silicose nos trabalhadores expostos.

Algumas iniciativas ligadas ao controle da exposição ocupacional à sílica vêm sendo desenvolvidas desde $1990^{26}$. Campanhas nacionais para redução da exposição foram iniciadas pelos sindicatos dos trabalhadores em 1992. Legislações estaduais proibindo o uso de areia como abrasivo do jatea- mento foram adotadas no Estado do Rio de Janeiro em $1992^{6}$ e a seguir, em 1997, pelo Estado do Paraná e pela cidade de Joinville. Em 2002 teve início o "Programa Nacional de Eliminação da Silicose”, por meio de convênio de cooperação entre os Ministérios da Saúde, Ministério do Trabalho e Emprego, representantes do patronato industrial e dos trabalhadores ${ }^{26,27}$. Em 2004, o uso de areia como abrasivo, em qualquer atividade econômica, foi proibido nacionalmente pela Portaria 99 do Ministério do Trabalho e Emprego.

A MEO elaborada para este estudo permitiu dimensionar o número de trabalhadores exposto à sílica no Brasil e identificar os setores com a prevalência mais alta. Grupos definidos de trabalhadores mais intensamente expostos à sílica foram localizados. Assim, já é possível priorizar o registro sistemático de trabalhadores expostos e desencadear legislações e atividades de vigilância, especialmente nos setores onde alternativas tecnológicas são conhecidas e disponíveis ${ }^{28}$.

\section{Referências}

1. NIOSH. Health Effects of Occupational Exposure to Respirable Crystalline Silica. Cincinnati: DHHS (NIOSH) Publication 129; 2002.

2. IARC monographs on the evaluation of carcinogenic risks to humans. Silica, some silicates, coal dust and paraaramid fibrils. IARC 1997; 68: 41-242.

3. Kauppinen T, Toikkanen J, Pedersen D, Young R, Ahrens $\mathrm{W}$, Boffetta $\mathrm{P}$, et al. CAREX. International Information System on Occupational Exposure to Carcinogens [MS Access database on line]. Finland: Finish Institute of Occupational Health; 1998a. Disponível em URL:http:/ / www.occuphealth.fi/list/data/CAREX/ [Acessado em 3 de outubro de 2006].

4. Algranti E. Occupational lung diseases in Brazil. In: Banks DE, Parker JE (Eds). Occupational Lung Disease - An International Perspective. London: Chapman \& Hall Medical; 1998. p 105-15.

5. Algranti E. Epidemiologia das Doenças Ocupacionais Respiratórias no Brasil. In: Menezes AMB. Epidemiologia das Doenças Respiratórias - Série Pneumologia Brasileira. Rio de Janeiro: Revinter; 2001. p 119-43.
6. Castro HA, Bethlem EP. A silicose na indústria naval do Estado do Rio de Janeiro: análise parcial. Jornal de Pneumologia 1995; 21: 13-6.

7. Holanda MA, Holanda MA, Martins MP, Felismino PH, Pinheiro VG. Silicosis in Brazilian pit diggers: relationship between dust exposure and radiological findings. $\mathrm{Am} \mathrm{J}$ Ind Med 1995; 27: 367-78.

8. Antão VC, Pinheiro GA, Kavakama J, Terra-Filho M. High prevalence of silicosis among stone carvers in Brazil. Am J Ind Med 2004; 45: 194-201.

9. Oliveira JI. Prevalence of silicosis among ceramic industry workers in the city of Pedreira, Brazil. Abstracts of communications. VII International Pneumoconiosis Conference; 1998: 114.

9. Mendonça EMC, Silva RCC, Algranti E, Bussacos MA. Respiratory impairment in Brazilian foundry workers exposed to sand. Medicina del Lavoro 2002; 93: S28

10. Ribeiro FSN, Parreiras M, Algranti E, Handar Z, Bedricow B. A Dimensão da Silicose na Indústria da Construção Civil Brasileira. Recife: V Congresso Nacional sobre Condições e Meio Ambiente de Trabalho na Indústria da Construção dos Países do Mercosul; 2005: 203-7. 
12. IBGE. Demográfico de Censo: Divulgação de resultados 2002. [consecutivo on-line]. Disponível em http:// www.ibge.gov.br/home/default.php [Acessado em 1 de agosto de 2004].

13. Ribeiro FSN, Wünsch Filho V. Avaliação retrospectiva da exposição ocupacional a cancerígenos: abordagem epidemiológica e aplicação em vigilância em saúde Cad Saúde Pública 2004; 20: 881-90.

14. Siemiatycki J. Risk Factors for Cancer in the Workplace, Florida: CRC Press; 1991.

15. Ribeiro FSN, Camargo EA, Wünsch Filho V. Delineamento e validação de matriz de exposição ocupacional à sílica. Rev Saúde Pública 2005; 39: 18-26.

16. Burgess GL. Development of an exposure matrix for respirable crystalline silica in the British pottery industry. Ann Occup Hyg 1998; 42: 209-17.

17. Kauppinen, T.; Toikkanen, J.; Pedersen, D.; Young, R.; Ahrens, W.; Boffetta, P.; Hansen, J. et al. Occupational exposure to carcinogens in European Union. Occup Environ Med 2000; 57: 10-8.

18. Sanderson WT, Steeland K, Deddens J. Historical respirable quartz exposures of industrial sand workers: 1946-1996. Am J Ind Med 2000; 38: 389-98.

19. Rybicki BA, Johnson CC, Peterson EL, Kortsha GX, Gorell JM. Comparability of different methods of retrospective exposure assessment of metals in manufacturing industries. Am J Ind Med 1997; 31: 36-43

20. Mirabelli D. Estimate of the number of workers exposed to cancer causing agents in Italy, within the framework of European study CAREX. Epidemiologia i Prevenzione 1999; 23: 346-59.
21. Kauppinen, T, Toikkanen J, Pukkala E. From crosstabulations to multipurpose exposure information system: a new job-exposure matrix. Am J Ind Med 1998; 33: 409-17.

22. MTE (Ministério do Trabalho e Emprego). Registros Administrativos Rais e Caged. Brasília: MTE/SPES/ CGETIP; 1999.

23. Fundacentro. Levantamento das condições de higiene segurança do trabalho, Mineração Lapa Vermelha Ltda. Pedro Leopoldo/MG, Projeto Pedreiras [Relatório do estudo das condições de higiene e segurança do trabalho, Mineração Lapa Vermelha Ltda., Pedro Leopoldo/MG, Projeto Pedreiras]. São Paulo: MTE/Fundacentro; 1985.

24. Souza VF, Quelhas OLG. Avaliação e controle da exposição ocupacional à poeira na indústria da construção. Ciência \& Saúde Coletiva 2003; 8: 801-7.

25. NIOSH. Work-Related Lung Disease Surveillance - Report 1996. Publication No 96-134. Disponível em http:// www.cdc.gov/niosh/w7_pref.html [Acessado em 30 de julho de 2004].

26. Algranti E, Handar Z, Ribeiro FSN, Bon AMT, Santos AM, Bedricow B. Exposición a sílice y silicosis en el Programa Nacional de Eliminación de Silicosis en Brasil (PNES). Ciencia y Trabajo 2004; 6: 1-13.

27. Goelzer B, Handar Z. Programa Nacional de Eliminação da Silicose. 2002. Disponível em http:// www.fundacentro.gov.br/sílicaesilicose [Acessado em 20 de novembro de 2004].

28. Brasil. Portaria n.99 de 19 de outubro de 2004 do Ministério do Trabalho e Emprego. Proíbe o processo de trabalho de jateamento que utilize areia seca ou úmida como abrasivo. Publicado no Diário Oficial da União, em 21 de outubro de 2004. 